\title{
Une nouvelle forme de potentialisation à long terme au niveau de I'hippocampe
}

Un des problèmes les plus fascinants en neurobiologie concerne les mécanismes synaptiques qui contribuent aux processus de mémorisation et d'apprentissage. Au début de ce siècle, Cajal, en 1911 [1], puis Hebb en 1949 [2], avaient proposé que mémorisation et apprentissage résultaient de changements, liés à un usage répété, dans la "puissance " de la transmission synaptique. Cette hypothèse fut tout d'abord testée dans la moelle épinière, mais le résultat se solda par un échec puisqu'une stimulation à haute fréquence des afférences de type $\mathrm{I}_{\mathrm{A}}$ n'augmentait la réponse post-synaptique des motoneurones que pendant quelques minutes [3]. En 1973, Bliss et Lomo [4] montrèrent qu'une stimulation de haute fréquence et de brève durée $(100 \mathrm{~Hz}$ pendant 3 à 4 secondes ou $10 \mathrm{~Hz}$ pendant 10 à 15 secondes) des afférences excitatrices de l'hippocampe, entraînait une augmentation persistante de la réponse postsynaptique allant de plusieurs heures, in vitro, à plusieurs jours, voire des semaines sur l'animal entier. Cette augmentation persistante de l'efficacité synaptique fut appelée " potentialisation à long terme " (PLT). La durée de ce phénomène mais aussi ses propriétés d'associativité et de spécificité font de la PLT le modèle expérimental le plus étudié, à l'heure actuelle, du processus mnésique $(\mathrm{m} / \mathrm{s}$ $n^{\circ} 3$, vol. 6, p. 295). Les études sont le plus souvent effectuées au niveau de la région dite CA1 de l'hippocampe (ou secteur de Sommer chez l'homme) dont la dégénérescence est associée, chez l'homme comme chez l'animal, à des défauts mnésiques importants [5].

Il est maintenant clairement admis que l'induction de la PLT au niveau de CA1 est contrôlée par le récepteur NMDA (N-méthyl D-aspartate), un sous-type de récepteur au glutamate qui est le principal neurotransmetteur des afférences excitatrices de l'hippocampe. Le canal associé au récepteur NMDA est perméable notamment aux ions $\mathrm{Ca}^{++}$. Au potentiel de repos, ce canal est bloqué par les ions magnésium de façon dépendante du voltage [6], de telle sorte qu'une dépolarisation importante du type de celle produite par un train de stimulation, ôte ce blocage et l'augmentation $\mathrm{du}\left(\mathrm{Ca}^{++}\right)$intracellulaire qui en résulte constitue un élément clé de la cascade d'événements qui vont se traduire par une potentialisation persistante de l'efficacité synaptique [7, 8]. Étant donné le rôle crucial du $\left(\mathrm{Ca}^{+}\right)$i dans la PLT, nous avons voulu voir si l'on pouvait induire une PLT en augmentant le $\left(\mathrm{Ca}^{++}\right) \mathrm{i}$ par d'autres voies que l'activation canalrécepteur NMDA. En effet, les cellules nerveuses possèdent des densités relativement importantes de différents types de canaux calciques dits " voltage-dépendant ", parce qu'ils ne sont activés que dans une plage donnée de potentiel de membrane. L'activation de ces canaux provoque une entrée rapide et massive du $\mathrm{Ca}^{++}$(à cause du gradient de concentration du $\mathrm{Ca}^{++}$respectivement $10^{-7} \mathrm{M}$ à l'extérieur et à l'intérieur de la cellule et donc un accroissement du $\left[\mathrm{Ca}^{++}\right]$i. Dans des concentrations physiologiques, ces canaux $\mathrm{Ca}^{++}$ sont relativement peu actifs du fait d'une multitude de canaux perméables au $\mathrm{K}^{+}$dont l'activation produit une hyperpolarisation et, ainsi, une "inhibition" de l'excitabilité cellulaire.

Neuf courants potassiques ont été décrits dans l'hippocampe; ils sont dénommés $I_{A}, I_{D}, I_{M}, I_{Q}, I_{f R}, I_{K}$, $I_{C}, I_{A H P}, I_{K A T P}$. Ces courants ont été différenciés selon trois critères : (1) la marge de potentiels membranaires où ils sont activés et inactivés ; (2) leurs cinétiques d'activation et d'inactivation ; (3) leur sensibilité pharmacologique. Cet éventail de différents types de canaux $\mathrm{K}^{+}$reflète le spectre important de fonctions dans lesquelles ces canaux sont impliqués (modulation de l'excitabilité cellulaire, repolarisation après un potentiel d'actions ou une bouffée de potentiels). Notre stratégie [9] a donc été d'étudier les effets des différents bloquants de ces courants potassiques sur la transmission synaptique dans la région CA1 de l'hippocampe à l'aide d'enregistrements intra- et extracellulaire. Nous avons ainsi constaté que le blocage de $\mathrm{I}_{\mathrm{C}}, \mathrm{I}_{\mathrm{M}}$ et $I_{K}$ par le TEA (tétraéthylammonium) à des concentrations supérieures à $15 \mathrm{mM}$, produisait une augmentation persistante de la réponse post-synaptique, alors que le blocage uniquement de $\mathrm{I}_{C}$ et $\mathrm{I}_{\mathrm{M}}$ ne produit qu'une potentialisation transitoire de la réponse; de même le blocage de $I_{A}$ et $I_{D}$ par le 4-aminopyridine ne produit pas de PLT. La PLT produite par de fortes concentrations de TEA, qui est due à une augmentation réelle de l'efficacité synaptique, n'est pas liée à une activation des récepteurs NMDA puisque l'application de l'APV, un antagoniste spécifique de ce type de récepteur ne bloque pas l'effet du TEA. En revanche, l'application du CNQX, un antagoniste sélectif des récepteurs non NMDA, ou de la flunarizine, un bloquant des canaux calciques "voltage dépendant ", empêchent le développement de la PLT. Ces données suggèrent 
que cette PLT est engendrée par une augmentation transitoire de la libération de glutamate qui en agissant sur les récepteurs non NMDA va induire une dépolarisation et permettre l'activation des canaux calciques voltage dépendant, processus qui est facilité par le blocage des courants potassiques (figure 1).

Ainsi nous montrons que l'augmentation de calcium interne produite par d'autres mécanismes que l'activation de récepteurs NMDA peut engendrer une PLT. On peut penser d'ailleurs qu'une augmentation du $\left(\mathrm{Ca}^{++}\right)$i produite par la troisième grande voie - une libération du $\left(\mathrm{Ca}^{+}\right)$à partir des stores de stockage intracellulaire par exemple sous l'effet de la caféine - va également engendrer une PLT. Il reste à mieux comprendre les mécanismes qui

Figure 1. Mise en évidence de deux types de potentialisation à long terme dont l'induction implique deux mécanismes différents. A. Dans les conditions normales d'activation, le glutamate libéré par chaque potentiel d'action active essentiellement les récepteurs AMPA. Les canaux calciques "voltagedépendant " ainsi que les récepteurs canaux NMDA sont relativement peu activés. B. Potentialisation à long terme (PLT) dépendante des récepteurs NMDA. La sommation temporelle des dépolarisations rapides produite par le glutamate via les récepteurs AMPA pendant une stimulation de haute fréquence permet l'activation du récepteur NMDA en débloquant le canal; il s'en suit un flux entrant d'ions calcium à travers ce canal qui va produire une potentialisation à long terme. C. Potentialisation à long terme indépendante des récepteurs NMDA. Le blocage des courants potassiques par le TEA, augmente la durée du potentiel d'action présynaptique et, en conséquence, produit une large augmentation de la libération de glutamate. Celui-ci, en agissant sur les récepteurs AMPA, entraîne une large dépolarisation post-synaptique qui permet l'activation de courants calciques "voltage-dépendant" dont l'effet sera l'induction d'une potentialisation à long terme.

$\mathrm{m} / \mathrm{s} n^{\circ} 1$, vol. 7 , janvier 91
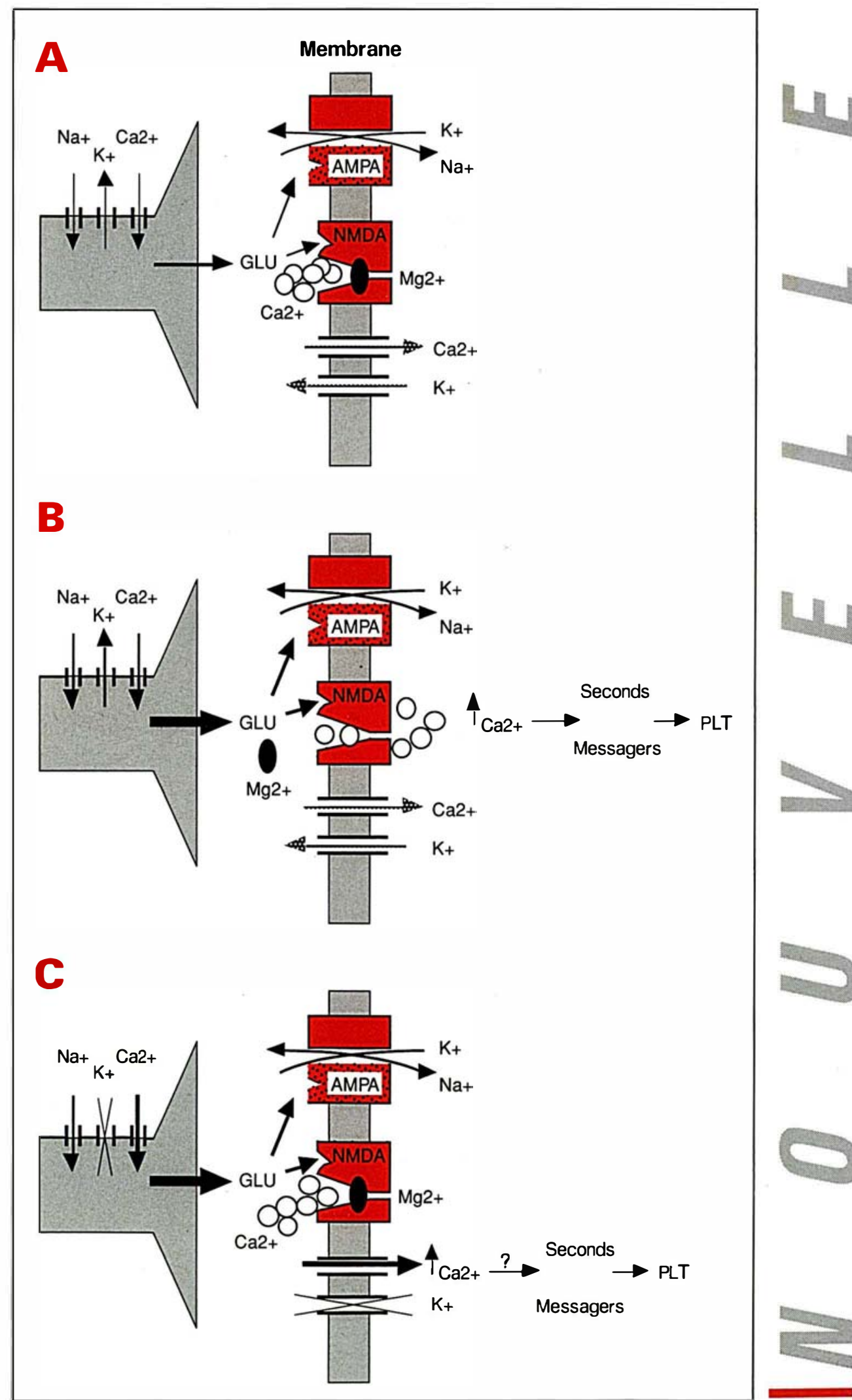
contrôlent le $\left[\mathrm{Ca}^{++}\right] \mathrm{i}$ et l'expression de telle ou telle forme de PLT. On peut par exemple envisager que les PLT relayées par les récepteurs NMDA et par les canaux calciques "voltage dépendant " seraient contrôlées respectivement par les inhibitions impliquant les récepteurs GABA et celles impliquant les canaux $\mathrm{K}^{+}$ "voltage-dépendant ". Le fait que la pharmacologie des canaux $\mathrm{K}^{+}$soit en plein essor ouvre des perspectives intéressantes pour mieux définir le rôle de ces canaux dans l'intégration neuronale et les effets de bloquants sélectifs dans la fonction mnésique [10].

L.A.

Y.B.A.

1. Cajal SR. Histologie du système nerveux de l'homme et des vertébrés. 1911; 2 : 993.

2. Hebb DO. The organization of behavior. New York: John Wiley and Sons Inc, 1949.

3. Spencer WA, April RS. Plastic properties of monosynaptic pathways in mammals. In : Horn G, Hinde RA, eds. Short term changes in neuronal activity and behavior. Cambridge : Cambridge university press, 1970 : 433-74.

4. Bliss TVP, Lomo T. Long-lasting potentiation of synaptic transmission in the dentate area of the anaesthetized rabbit following stimulation of the perforant path. J Physiol 1973 ; 232 : 331-56.

5. Zola-Morgan S, Squire LR, Amaral DG. Human amnesia and the medial temporal region : enduring memory impairement following a bilateral lesion to field CA1 of the hippocampus. I Neurosci 1986 ; 6 : 2950-67.

6. Nowak L, Bregestowski P, Ascher P, Herber A, Prochiantz A. Magnesium gates glutamate-activated channels in mouse central neurones. Nature 1984; 307 : 462-5.

7. Collingridge GL, Bliss TVP. NMDA receptors, their role in long-term potentiation. Trends Neurosci 1987 ; 10 : 288-93.

8. Malenka RC, Kauer J, Perkel DJ, Nicoll RA. The impact of postsynaptic calcium on synaptic transmission : its role in long term potentiation. Trends Neurosci 1989 ; 12 : 444-50. 9. Aniksztejn L, Ben-Ari Y. A novel form of long term potentiation produced by a $\mathrm{K}^{+}$ channel blocker in the hippocampus. Nature 1991 ; 349 : 67-9.

10. Cherubini E, Ben-Ari Y, Gho M, Bidart JN, Lazdunski M. Long term potentiation of synaptic transmission in the hippocampus induced by a bee venom peptide. Nature $1987 ; 328: 70-3$ 